\title{
Consecutive ones property testing: cut or swap
}

\author{
Mathieu Raffinot ${ }^{1}$ \\ LIAFA, Univ. Paris Diderot - Paris 7, 75205 Paris Cedex 13, France. \\ raffinot@liafa.jussieu.fr
}

\begin{abstract}
Let $\mathcal{C}$ be a finite set of $n$ elements and $\mathcal{R}=\left\{R_{1}, R_{2}, \ldots, R_{m}\right\}$ a family of $m$ subsets of $\mathcal{C}$. The family $\mathcal{R}$ verifies the consecutive ones property if there exists a permutation $P$ of $\mathcal{C}$ such that each $R_{i}$ in $\mathcal{R}$ is an interval of $P$. There already exist several algorithms to test this property in $O\left(\sum_{i=1}^{m}\left|R_{i}\right|\right)$ time, all being involved. We present a simpler algorithm, based on a new partitioning scheme.
\end{abstract}

\section{Introduction}

Let $\mathcal{C}=\left\{c_{1}, \ldots, c_{n}\right\}$ be a finite set of $n$ elements and $\mathcal{R}=\left\{R_{1}, R_{2}, \ldots, R_{m}\right\}$ a family of $m$ subsets of $\mathcal{C}$. Those sets can be seen as a 0-1 matrix, where the $\mathcal{C}$ represents the columns and each $R_{i}$ the ones of row $i$. Figure 1 shows such a matrix.

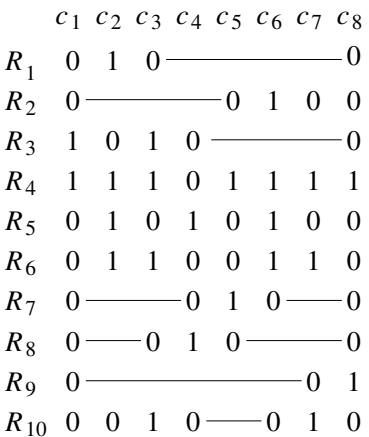

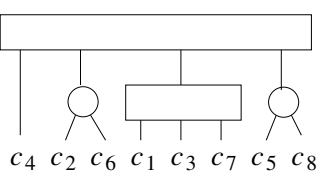

PQ-tree

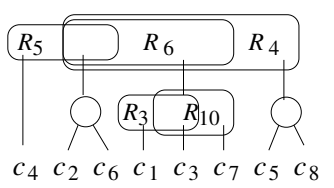

Overlap classes

Fig. 1. A matrix verifying the consecutive ones property, its associated PQ-tree and the information contained in overlap classes. In the PQ-tree, $Q$ nodes are represented by boxes, while $P$ nodes by circles.

The family $\mathcal{R}$ verifies the consecutive ones property (C1P) if there exists a permutation $P$ of $\mathcal{C}$ such that each $R_{i}$ in $\mathcal{R}$ is an interval of $P$. For instance, the family given by the matrix of 1 verifies $\mathrm{C} 1 \mathrm{P}$. Efficiently testing $\mathrm{C} 1 \mathrm{P}$ has received a lot of attention in the literature for this problem to be strongly related to the recognition of interval graphs, the recognition of planar graphs, modular decomposition and others graph decompositions. The consecutive ones property is the core or many other algorithms that have applications in a wide range 
of domains, from VLSI circuit conception through planar embeddings [10 to computational biology for the reconstruction of a chromosome from a set of contigs [3]. We denote $|\mathcal{R}|=\sum_{i=1}^{m}\left|R_{i}\right|$. Several $O(|\mathcal{R}|)$ time algorithms have been proposed to test this property, following five main approaches.

The first approach and still the most well known one is the use of PQ-tree structure [1. A PQ-tree is a tree that represents a set of permutations defined by the possible orders of its leaves obtained by changing the order of the children of any internal node depending of its type which can be $P$ or $Q$. For a $P$ node, any order of its children is valid, while for a $Q$ node only the complete reversal of its children is accepted. For instance, in Figure 1, the PQ-tree represents the order $c_{4} c_{2} c_{6} c_{1} c_{3} c_{7} c_{5} c_{8}$, but also $c_{4} c_{2} c_{6} c_{7} c_{3} c_{1} c_{5} c_{8}, c_{4} c_{6} c_{2} c_{7} c_{3} c_{1} c_{8} c_{5}$, and so on. The main point for using PQ-trees is that if a family verifies C1P, then one can build a PQ-tree representing exactly all column orders for which the C1P will be verified. For instance, the PQ-tree in Figure 1 represents all orders for which the family given by the matrix at its right verifies C1P. If a family does not verify $\mathrm{C} 1 \mathrm{P}$, its associated PQ-tree is said empty.

Given a family, in order to build its associated PQ-tree, each row is inserted one after the other in the tree while the PQ-tree is not empty. This update is done through a procedure called Refine which complexity is amortized on the size of the tree. The main drawback of this approach is that the implementation of Refine in its linear time complexity is still a challenge. It uses a series of 11 templates depending on the form of the tree and choosing which to use in constant time is a huge programming difficulty, that has only slightly been reduced by Young [11] using a recursive Refine that allows us to reduce the number of templates. Moreover, extracting a certificate that the family does really not verify C1P from this approach is hard. Therefore, given a PQ-tree implementation, one can hardly be confident neither in its validity nor in its time complexity. This is the reason why many other algorithmic approaches have been tempted to test C1P using simpler and/or certified algorithms.

One of those attempt consists in first transforming the $\mathrm{C} 1 \mathrm{P}$ testing problem to interval graph recognition by adding fake rows and then use a special LexBFS traversal that produces a first order on $C$ that has some special properties [5]. A recursive partitioning phase is then necessary following both this LexBFS order and an order on the rows derived from a clique tree built from the LexBFS traversal. This approach is also complex, both to understand and to program, and surprisingly the links between these two first approaches are not that clear.

A third approach was to try to design the PC-tree [8], an easiest structure to refine than the PQ-tree. However as Haeupler and Tarjan noticed in [6], the authors of [8] did not consider "implementations issues" (sic) than lead to incorrect algorithms for $\mathrm{C} 1 \mathrm{P}$ testing and planar graph recognition.

A fourth approach appeared in [7] with the idea of simplifying the C1P test by avoiding PQ-tree. However, the algorithm remains very involved.

A last and more recent approach has been presented by R. McConnell in 9 . This approach is a breakthrough in the understanding of the intrinsic constraints of $\mathrm{C} 1 \mathrm{P}$ and the real nature of the $\mathrm{PQ}$-tree. We describe this approach in details 
since our method is a tricky simplification of it. McConnell shows that each $Q$ node of the PQ-tree represents in fact an overlap class of a subset of the rows. Two rows $R_{i}$ and $R_{j}$ of $\mathcal{R}$ overlap if $R_{i} \cap R_{j} \neq \emptyset, R_{i} \backslash R_{j} \neq \emptyset$, and $R_{j} \backslash R_{i} \neq \emptyset$. An overlap class is a equivalence class of the overlap relation, that is, two rows $R_{i}$ and $R_{j}$ are in the same class if there is a chain of overlaps from $R_{i}$ to $R_{j}$. For instance, the two non trivial overlap classes of the family example given by the matrix of Figure 1 are shown on the same figure on the right. Overlap classes partition the set of rows and form a laminar family, and thus they can be organized in an inclusion tree.

This tree is the skeleton of the PQ-tree and the remaining $P$ node might also been derived from the overlap classes. However, for an equivalence class to be a node of the PQ-tree, it also has to verify the consecutive one property. Thus, where is the gain ? The trick used by McConnell is that verifying the $\mathrm{C} 1 \mathrm{P}$ of an overlap class is independent of the other overlap classes and somehow easier provided a spanning tree of the overlap graph of the class. Using a partitioning approach guided by this tree, it is linear in the total size of the rows in an overlap class to test if this overlap class verifies $\mathrm{C} 1 \mathrm{P}$. Consequently, by testing overlap classes one after the other, one can verify if the whole set $\mathcal{R}$ fulfills $\mathrm{C} 1 \mathrm{P}$ in $O(|\mathcal{R}|)$ time. The technical complexity of the approach is twofold: (a) compute overlap classes and (b) a spanning tree of each class.

Point (a) is performed in 9 through an algorithm of Dahlhaus published as a routine of [4] used for undirected graph split decomposition. It is considered by McConnell as a black box that takes as input $\mathcal{R}$ and returns a list of overlap classes and for each overlap class the list of rows that belongs to.

Point (b) is then computed in [9] for each overlap class by a complex add-on from the list of rows in the class.

In this article we present a simplification of this last approach by introducing a new partitioning scheme. It should be noted first that McConnell's approach can already be very slightly simplified using existing tools. Indeed, the algorithm of Dahlhaus for computing overlap classes is an algorithmic pearl that has been recently simplified and made computationable in the sense that the original version uses an LCA while the simplified version presented in 2] only uses partitioning. Moreover, a modification of Dahlhaus's approach allows us to extract a spanning tree of each overlap class. This modification is not obvious but remains simpler than the add-on of $[9$. However, building a spanning tree from Dahlhaus is intrinsically difficult, because the two concepts are somehow antinomic: Dahlhaus's approach maintains some ambiguities in the row overlaps that permit to gain on the overall computation, while computing a spanning tree requires solving most of these ambiguities, which is sometimes difficult. In this paper, we successfully maintain these ambiguities even in the partitioning phase, avoiding buliding a spanning tree.

To clearly present our approach let us consider the difference between the PQ-tree approach and that of McConnell in terms of partitioning. The PQ-tree records a partition of $\mathcal{C}$ induced by the rows even if some rows can be included in others (a row might not cut any class of the partition). The difficulty arises when 
updating the structure: in the same time we need to update both a partition and an inclusion tree that are intrinsically merged. In the second approach the idea is to impose that each row added surely overlaps a previous one, which simplifies the partitioning since the inclusion tree as not to be maintained. This also insures the linear time complexity without any amortizing need, but at the cost of the computation of a spanning tree of each overlap class.

Our approach lies in between. For each overlap call we update a partition, but we also allow some fail and swap in the partitioning scheme. We compute an order that guaranties that when adding a new row $R_{1}$, if it does not overlap any row already considered, then the row following $R_{2}$ will, and moreover $R_{1}$ overlaps $R_{2}$ and will be considered next. We thus swap $R_{1}$ and $R_{2}$ in the order we update the partition if $R_{1}$ does not cut. We call this order a "swap overlap order". This order could of course be obtained from a spanning tree, but we explain below how we can compute such an order at a very small computational price by entering deeper in Dahlhaus's algorithm, that we also slightly simplify for our needs. Our algorithm thus runs in 3 main steps: (1) the computation of each overlap class using an algorithm close to that of Dahlhaus, (2) for each class we compute of a swap overlap order, and (3) we partition each class guided by this order using a new partitioning scheme. If the partitioning fails on a class, the $\mathrm{C} 1 \mathrm{P}$ is not verified. Steps 1 and 2 are performed in the same time, but for clarity we present them in two distinct steps.

This article is organized as follows. In the following Section 2 we present two variations of Dahlhaus's algorithm for computing overlap classes. In Section 3 we explain our main notion of swap overlap order and explain how to slightly modify Dahlhaus's algorithm to generate such an order for each overlap class. In Section 4 we eventually explain how to test $\mathrm{C} 1 \mathrm{P}$ on each overlap class using the swap overlap order associated to. We added two appendixes. The first is an example of the construction of a swap overlap order. The second is a technical routine used in Dahlhaus's algorithm revisited in [2] that we mainly recall.

\section{Computing Overlap Classes}

In this section we recall and slightly modify the algorithm of Dahlhaus for computing overlap classes already simplified and presented in 2]. The computational problem to efficiently compute the overlap classes comes from the fact that the underlying overlap graph, where $R_{i}$ are the vertices and $\left(R_{i}, R_{j}\right)$ is an edge if $R_{i}$ overlaps $R_{j}$, might have $\Theta\left(|\mathcal{R}|^{2}\right)$ edges and thus be quadratic in $O(|\mathcal{R}|)$. An overlap class is a connected component of this graph.

Let LR be the list of all $R \in \mathcal{R}$ sorted in decreasing size order. The ordering of sets of equal size is arbitrarily fixed, and thus LR is a total order. Given $R \in \mathcal{R}$, we denote $\operatorname{Max}(R)$ as the largest row $X \in \mathcal{R}$ taken in $L R$ order such

that $X<_{L R} R$ and $X$ overlaps $R$. This definition is modified from that in [2] to consider the order LR in the definition of $\operatorname{Max}(R)$. 
Note that $\operatorname{Max}(R)$ might be undefined for some sets of $\mathcal{R}$. In this latter case, in order to simplify the presentation of some technical points, we write $\operatorname{Max}(R)=\emptyset$. Dahlhaus's algorithm is based on the following observation:

Lemma 1 ([4]2]). Let $R \in \mathcal{R}$ such that $\operatorname{Max}(R) \neq \emptyset$. Then for all $X \in \mathcal{R}$ such that $X \cap R \neq \emptyset$ and $|R| \leq|X| \leq|\operatorname{Max}(R)|, X$ overlaps $R$ or $\operatorname{Max}(R)$.

The trick we propose below for computing the overlap order of each overlap class is also based on lemma 1 .

Let us assume first that we already computed all $\operatorname{Max}(R)$. For each column $c \in \mathcal{C}$ we compute the list $S L(c)$ of all sets $R \in \mathcal{R}$ to which $c$ belongs. This list is sorted in increasing order of the sizes of the sets respecting LR, thus in decreasing order in LR. Computing and sorting all lists for all $c \in \mathcal{C}$ can be done in $O(|\mathcal{R}|)$ time using a stable bucket sort.

Dahlhaus's overlap class identification is built on those lists. For all $c \in \mathcal{C}$, let $R$ be a set containing $c$ such that $\operatorname{Max}(R) \neq \emptyset$. We define a new interval on $S L(c)$ beginning in $R$, continuing from $R$ in the order of $S L(c)$ and finishing by the greatest row in $S L(c)$ such that $|Y| \leq|\operatorname{Max}(R)|$. Notice that this greatest row $Y$ is not necessarily equal to $\operatorname{Max}(R)$. If it is the case, the interval is said of type $M$ (for Max included), of type $E$ (for External) otherwise. Given an interval $I, \operatorname{First}(I)$ is the first row of the interval, thus the row which generates the interval.

We "bucket" sort the intervals in a table $T I[1 . . m]$ of $m$ entries the following way. For an interval $I=\left[R_{i_{1}} \ldots R_{i_{k}}\right], I$ is added to all $T I\left[i_{j}\right], 1 \geq j \geq k$.

An example of a family and the intervals associated to is shown in figure 2 .

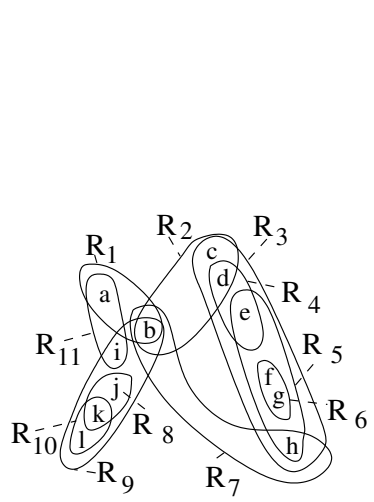

$$
\begin{aligned}
& \text { a } \overline{\mathrm{R}_{11}} \overline{\mathrm{R}}_{1}{ }^{\mathrm{E} 1} \\
& \text { b } \overline{\mathrm{R}}_{7}^{-} \overline{\mathrm{R}}_{1}^{-} \overline{\mathrm{R}}_{2}^{-} \overline{\mathrm{R}}_{9}^{-\mathrm{E} 2} \\
& \text { c } \overline{\mathrm{R}_{2} \mathrm{R}_{3}} \mathrm{M} 3 \\
& \text { d } \mathrm{R}_{4}-\overline{\mathrm{R}_{2} \mathrm{R}_{3}} \text { M5 } \\
& \text { e } \overline{\mathrm{R}_{4} \mathrm{R}_{5}} \mathrm{R}_{3}{ }^{\mathrm{M} 6} \\
& \text { f } \mathrm{R}_{6} \mathrm{R}_{5} \mathrm{R}_{3} \\
& \text { g } \mathrm{R}_{6} \mathrm{R}_{5} \mathrm{R}_{3} \\
& \text { h } \overline{\mathrm{R}_{7} \mathrm{R}_{5} \mathrm{R}_{3}} \mathrm{M} 7 \\
& \text { i } \quad \overline{\mathrm{R}}_{11} \mathrm{R}_{9} \mathrm{M} 8 \\
& \text { j } \quad \mathrm{R}_{8} \mathrm{R}_{9} \\
& { }_{\mathrm{k}}^{\mathrm{M} 9} \mathrm{R}_{10} \mathrm{R}_{8} \mathrm{R}_{9} \\
& \text { l } \mathrm{R}_{10} \mathrm{R}_{9}
\end{aligned}
$$

TI

E1 E2

M3 M5 E4 E2

M3 M5 M7

M6 E4

M6 M7

M7 E2

M9

M8 E2

M9

$1 \quad$ M8 E1

Fig. 2. Example: a family $\mathcal{R}$, its corresponding sets $S L$ and the associated $T I$ table. Intervals of type $M$ are denoted by a plain line, while intervals of type $E$ are denoted by a dash one. 
To compute overlap classes, we mark them one after the other, keeping the numbering of the overlap class each row belongs to in a table $N C[1 . . m]$ all initialized to 0 .

\section{Algorithm 1: computing all overlap classes}

1. Initialize the counter $n c=1$ to count the overlap class we are tagging;

2. Choose an arbitrary $l, 1 \leq l \leq m$ such that there exist at least on interval in $T I[l]$;

3. For all interval(s) $I=\left[R_{i_{1}} \ldots R_{i_{k}}\right]$, in $T I[l]$.

(a) remove all occurrences of $I$ out of $T I$;

(b) mark each row in $I$ to belong to overlap class $n c$, thus $N C\left[i_{j}\right]=n c$, $1 \leq j \leq k$

(c) recurse this algorithm from step 3 on all $i_{j}, 1 \leq j \leq k$, such that $T I\left[i_{j}\right]$ is not empty;

(d) end the recursive procedure;

4. Increment $n c$ and apply step 2 while $T I[l]$ is not empty.

Rows that are not marked during this algorithm are themselves an overlap class of a single element that it is not necessary to consider further for testing C1P. We focus below on overlap classes that contain at least 2 rows.

By lemma 1. all rows in a given interval belong to the same overlap class. We prove now that Algorithm 1 computes all overlap classes.

First, assume that 2 rows $R_{i}$ and $R_{j}$ are such that $N C\left[R_{i}\right]=N C\left[R_{j}\right]$. Then the two rows have been marked during a recursive call of Step 3 that recurse on each interval containing a row. Thus the whole process computes the closure of belonging to a same interval, which guaranties that the two rows are linked by a chain of overlap(s).

Secondly, assume that two rows $R_{1}$ and $R_{2}$ overlap. Let us consider wlog that $R_{2}<_{L R} R_{1}$. Then $\operatorname{Max}\left(R_{1}\right)$ exists and as $R_{1}$ and $R_{2}$ intersect on at least one column $c, R_{2}$ is in an interval beginning in $R_{1}$ on $S L(c)$. We thus proved that:

Proposition 1 ([4]). Algorithm 1 computes all overlap classes of $\mathcal{R}$.

Worst case complexity of Algorithm 1. Algorithm 1 can be implemented to run in $O(|\mathcal{R}|$ ), provided that for a given row $R$ computing $\operatorname{Max}(R)$ is $O(1)$ time (see Appendix $B$ for details on this computation).

Up to now we dispose of a general scheme for computing all overlap classes of $\mathcal{R}$ that is directly adapted from 42 . We now modify this approach to consider the two types $M$ and $E$ of intervals successively for each row, beginning with intervals of type $M$ and then intervals of type $E$.

\section{Algorithm 2: the computation of all overlap classes revisited}

1. Initialize the counter $n c=1$ to count the overlap class we are tagging;

2. Choose an arbitrary $l, 1 \leq l \leq m$ such that there exist at least on interval in $T I[l]$ of type $M$; 
3. For all interval(s) $I=\left[R_{i_{1}} \ldots R_{i_{k}}\right]$ of type $M$ in $T I[l]$,

(a) remove all occurrences of $I$ out of $T I$;

(b) mark each row in $I$ to belong to overlap class $n c$, thus $N C\left[i_{j}\right]=n c$, $1 \leq j \leq k$

(c) recurse this algorithm from step 3 on all $i_{j}, 1 \leq j \leq k$, such that $T I\left[i_{j}\right]$ is not empty;

4. For all interval(s) $J=\left[R_{i_{1}} \ldots R_{i_{k}}\right]$ of type $E$ in $T I[l]$,

(a) remove all occurrences of $J$ out of $T I$;

(b) mark each row in $J$ to belong to overlap class $n c$, thus $N C\left[i_{j}\right]=n c$, $1 \leq j \leq k$

(c) recurse this algorithm from step 3 on all $i_{j}, 1 \leq j \leq k$, such that $T I\left[i_{j}\right]$ is not empty;

(d) end the recursive procedure;

5 . Increment $n c$ and apply step 2 while $T I[l]$ is not empty.

Algorithm 2 is still valid since (a) it is a simple modification of Algorithm 1 only considering two types of intervals and (2) in each overlap class there exist at least one interval of type $M$ to begin with at step 2 .

\section{Swap Overlap Order}

A swap overlap order is an order $R_{i_{1}} \ldots R_{i_{k}}$ on the rows of an overlap class such that, for all $2 \leq l \leq k$, at least one of the two following cases is true:

- $R_{i_{l}}$ overlaps one $R_{i_{g}}, 1 \leq g<l$,

$-l<k$ and $R_{i_{l+1}}$ overlaps $R_{i_{g}}, 1 \leq g<l$, and $R_{i_{l}}$ overlaps $R_{i_{l+1}}$.

We now modify Algorithm 2 to output for each overlap class a swap overlap order.

\section{Algorithm 3: outputing a swap overlap order for all overlap classes}

1. Initialize the counter $n c=1$ to count the overlap class we are tagging; Initialize $O_{n c}$ to the empty word $\epsilon$,

2. Choose an arbitrary $l, 1 \leq l \leq m$ such that there exist at least on interval in $T I[l]$ of type $M$;

3. For all interval(s) $I=\left[R_{i_{1}} \ldots R_{i_{k}}\right]$ of type $M$ in $T I[l]$,

(a) remove all occurrences of $I$ out of $T I$;

(b) concatenate to $O_{n c}$ successively the rows $R_{i_{1}}, R_{i_{k}}, R_{i_{2}}, \ldots, R_{i_{k-1}}$ in this order, adding a row only if $N C\left[i_{j}\right]=0$. After adding a row, change $N C\left[i_{j}\right]$ to no.

(c) recurse this algorithm from step 3 on all $i_{j}, 1 \leq j \leq k$, such that $T I\left[i_{j}\right]$ is not empty;

4. For all interval(s) $J=\left[R_{i_{1}} \ldots R_{i_{k}}\right]$ of type $E$ in $T I[l]$,

(a) remove all occurrences of $J$ out of $T I$;

(b) recurse step 3 on $T I\left[i_{1}\right]$; 
(c) concatenate to $O_{n c}$ successively the rows $R_{i_{2}}, R_{i_{3}}, . ., R_{i_{k}}$ in this order, adding a row only if $N C\left[i_{j}\right]=0$. After adding a row, change $N C\left[i_{j}\right]$ to no.

(d) recurse this algorithm from step 3 on all $i_{j}, 1<j \leq k$, such that TI $\left[i_{j}\right]$ is not empty;

(e) end the recursive procedure;

5 . Increment $n c$ and apply step 2 while $T I[l]$ is not empty.

The main difference with Algorithm 2 in terms of recursive call is step 4.(b), where we first recurse on First $(J)$ when considering an interval of type $E$ before processing the interval itself.

A trace of the execution of Algorithm 3 is given in Appendix A. For the largest overlap class of our current example, it returns the swap overlap order $O_{1}=R_{2} R_{3} R_{4} R_{5} R_{7} R_{1} R_{9} R_{11}$.

What is the idea behind algorithm 3 ? We begin an order by considering and interval of type $M$, say $I=\left[R_{i_{1}} \ldots R_{i_{k}}\right]$. By placing $R_{i_{1}}$ and then $R_{i_{k}}=$ $\operatorname{Max}\left(R_{i_{1}}\right)$ before all other rows in $I$, Lemma 1 guaranties that the following rows in $I$ overlap either $R_{i_{1}}$ or $R_{i_{k}}$.

Then, assume that there exits a row $X$ between $R_{i_{1}}$ and $R_{i_{k}}$ in $I$. We recurse on $X$. If the line corresponding to $X$ in $T I$ contains and interval, say $I^{\prime}=\left[R_{i_{1}}^{\prime} \ldots R_{i_{k^{\prime}}}^{\prime}\right]$, it be of two types, $M$ or $E$.

Case 1. If $I^{\prime}$ is of type $M$ then it will be process first before all type $E$ intervals corresponding to $X$. Then, either $X$ is the fist row of the interval, either not. Whatever, as $X$ already appears in $O_{n c}$ by interval $I$, then by concatenating the rows in the order $R_{i_{1}}^{\prime} R_{i_{k^{\prime}}}^{\prime} \ldots$ if not already in $O_{n c}$, we guaranty that:

- one of $R_{i_{h^{\prime}}}^{\prime}=\operatorname{Max}\left(R_{i_{1}}^{\prime}\right)$ or $R_{i_{1}}^{\prime}$ overlaps $X$ that is already placed in $O_{n c}$ by Lemma 1

- each following row in $I^{\prime}$, if any, either overlaps $R_{i_{k^{\prime}}}^{\prime}$ or $R_{i_{1}}^{\prime}$, or already appears in $O_{n c}$.

Case 2. If $I^{\prime}$ is of type $E$, then $R_{i_{k}^{\prime}}^{\prime}$ is not $\operatorname{Max}\left(R_{i_{1}}^{\prime}\right)$. Thus there is not guaranty that $\operatorname{Max}\left(R_{i_{1}}^{\prime}\right)$ (that has to exist since $I^{\prime}$ is an interval beginning in $R_{i_{1}}^{\prime}$ ) has already been placed in $O_{n c}$. Thus we first recurse on $R_{i_{1}}^{\prime}$ (step 4-(a)) to guaranty that after some recursion the rows $R_{i_{1}}^{\prime}$ and $\operatorname{Max}\left(R_{i_{1}}^{\prime}\right)$ appear somewhere in $O_{n c}$ before processing $I$. Then, by lemma 1 , each row following $R_{i_{1}}^{\prime}$ in $I^{\prime}$ overlaps either $\operatorname{Max}\left(R_{i_{1}}^{\prime}\right)$ or $R_{i_{1}}^{\prime}$. As both are already in $O_{n c}$, we simply concatenate them to $O_{n c}$ in step $4-(\mathrm{c})$.

Thus, summarizing the 2 cases, when concatenating new rows to $O_{n c}$, we can insure that either (a) we add a couple $(X, \operatorname{Max}(X))$, provided that at least one of those rows overlaps a row $Y$ already placed in $O_{n c}$ (note that if one of those rows is already in $O_{n c}$, then the result also holds), or (b) a row $X$ that surely overlaps a row already in $O_{n c}$. Using this approach we identify each overlap class 
and in the same time we build a swap overlap order for each overlap class.

Complexity. It is obvious that the time complexity is the same that Algorithm 1 or Algorithm 2, that is, $O(|\mathcal{R}|)$.

\section{Partitioning Each Overlap Class}

At this point, we built a swap overlap order for each non trivial overlap class. It remains to explain how to test $\mathrm{C} 1 \mathrm{P}$ on each such class using this order.

We use a partitioning that is relatively similar to that of $[9$, except that instead of being driven by a spanning tree it uses a swap overlap order that is easier to build since it is in the direct continuation of Dahlhaus's approach for computing overlap classes. However, the important difference is that using a swap overlap order we can not certify that we cut each time the current partition when refined by a new row. Instead, we can certify that if the new row $R_{1}$ does not cut, the following row $R_{2}$ will, and $R_{1}$ will then cut $R_{2}$. We thus swap the two rows in the partitioning.

Let us enter details. We maintain an ordered set of sets, called parts, of columns of $\mathcal{C}$. When adding a row, a part $C$ can only be cut in two parts $C^{\prime} C^{\prime \prime}$ such that $C^{\prime} \cup C^{\prime \prime}=C$ and $C^{\prime} \cap C^{\prime \prime}=\emptyset$. In the partitioning, $C$ is replaced by $C^{\prime} C^{\prime \prime}$ or $C^{\prime \prime} C^{\prime}$ depending the case, but the general order of the initial partition is maintained.

To begin the partitioning phase, we consider the first row $R_{i_{1}}$ of the overlap order $O_{n c}=R_{i_{1}} R_{i_{2}} \ldots R_{i_{k}}$ of overlap class $n c$. We create a first part in our partition $P_{1}$ that is composed of the columns of $R_{i_{1}}$. We then refine this partition with $R_{2}$ by first marking all elements of $R_{2}$. Suppose first that $R_{2}$ overlaps (or cuts) $R_{1}$ and let $X=R_{1} \cap R_{2}$. We partition $P$ by $R_{2}$ in $P_{2}=\left(R_{1} \backslash X\right)(X)\left(R_{2} \backslash X\right)$, thus we simply placed all common elements of $R_{1}$ and $R_{2}$ on a line in such a way that both $R_{1}$ and $R_{2}$ are intervals of $P$, which is the core of the C1P.

Let us now consider a new row $R_{i_{j}}$. We mark elements of $R_{i_{j}}$ in $P_{j-1}$. Suppose again that $R_{i_{j}}$ cuts a row already integrated to $P_{j-1}$. Let $Y$ be the set of elements of $R_{i_{j}}$ that already appear in $P_{j}$. Two cases may occur:

(a) if $Y=R_{i_{j}}$, we only try to group together the elements of $R_{3}$ in $P_{2}$. If we can, we only cut the parts accordingly to build $P_{j}$

(b) if $Y \neq R_{i_{j}}$, then we try to cluster the elements of $Y$ on a border (left or right) of $P_{j-1}$. If we can, we cut the parts accordingly and add a new part $\left(R_{i_{j}} \backslash Y\right)$ before (resp. after) all parts of $P_{j-1}$ if the border was the left (resp. right) one to eventually build $P_{j}$.

Example of partitioning on the first overlap class of our current data set with the order $R_{2} R_{3} R_{4} R_{5} R_{7} R_{3} R_{1} R_{9} R_{11}$. 


\begin{tabular}{l|l|c} 
Row & Columns & Partition \\
\hline$R_{2}$ & $\{b, c, d\}$ & (bcd) \\
$R_{3}$ & $\{c, d, e, f, g, h\}$ & (b)(cd)(efgh) \\
$R_{4}$ & $\{d, e\}$ & (b)(c)(d)(e)(fgh) \\
$R_{5}$ & $\{e, f, g, h\}$ & (b)(c)(d)(e)(fgh) \\
$R_{7}$ & $\{b, h\}$ & fail
\end{tabular}

The main point of this approach is that if this process fails for a given row, the overlap call does not verify $\mathrm{C} 1 \mathrm{P}$.

Proposition $2([\mathbf{9}])$. Let $R_{i_{1}} R_{i_{2}} \ldots R_{i_{k}}$ be a total order of the rows of a given overlap class nc such that each row $R_{i_{j}}, j>2$, overlaps a previous row $R_{i_{l}}, 1 \leq$ $l<j$. Then the above partitioning fails if and only if the overlap class nc does not verify $C 1 P$.

Proof. The intuition behind this theorem is that if two rows $R_{a}$ and $R_{b}$ overlap, the intersection $X=R_{a} \cap R_{b}$ must rely in between and the only two possible column orders respecting $\mathrm{C} 1 \mathrm{P}$ are $\left(R_{a} \backslash X\right)(X)\left(R_{b} \backslash X\right)$ or $\left(R_{b} \backslash X\right)(X)\left(R_{a} \backslash X\right)$.

Each part of the partition derives from the intersection of two rows or the difference of a row and its intersection with the other rows. Thus the order of the elements inside a part is not relevant and can be changed, but the global order of all parts is fixed and can not be changed (not considering a global reversal) without breaking the $\mathrm{C} 1 \mathrm{P}$ of the previous rows. This has for consequence that when adding a new row that overlaps (at least) one row that is already embedded in the current permutation, $\mathrm{C} 1 \mathrm{P}$ will be maintained only if the elements of the new rows can be embedded in $P$ respecting the order of its parts. The fact that the order of the elements inside each parts is not relevant allows us to split some parts (placed in the extremities of the touched zone) in two subparts, those touched by the new row on a side, the rest on the other side. This is the only operation authorized when adding a row to test if we can maintain C1P adding the new row.

A new row can be embedded in $P$ under those conditions only in the two cases (a) and (b) equivocated above. Therefore, if the partitioning is feasible, then the new partition "encodes" all possible column order for the set of rows considered up to this point to verify C1P. If not, this insures that no column order could be valid for the set of rows to verify C1P.

In our approach, as we manipulate swap overlap orders, the partitioning phase must be slightly modified in the following way. Suppose that we want to refine the partition $P_{j-1}$ with $R_{i_{j}}$. If $R_{i_{j}}$ does not overlap any previous row use in the partitioning, that is if all columns of $R_{i_{j}}$ either belong to the same part of $P_{j-1}$ of to none, we swap $R_{i_{j}}$ and $R_{i_{j+1}}$, refine the partition with $R_{i_{j+1}}$ and only then with $R_{i_{j}}$. The swap overlap order guaranties that $R_{i_{j+1}}$ will cut a previous row, and that $R_{i_{j}}$ overlaps $R_{i_{j+1}}$. We call this partitioning a swap partitioning.

Theorem 1. Let $R_{i_{1}} R_{i_{2}} \ldots R_{i_{k}}$ be a swap overlap order of the rows of a given overlap class nc. Then the above swap partitioning fails if and only if the overlap class nc does not verify C1P. 
Proof. By swapping the rows when necessary, we insure that the order of the $R_{i_{1}} R_{i_{2}} \ldots R_{i_{k}}$ rows in which we refine the partition verifies that each row $R_{i_{j}}, j>$ 2 , overlaps a previous row $R_{i_{l}}, 1 \leq l<j$, thus satisfying the conditions of proposition 2,

Implementation issues. Let us now consider the time complexity of our partitioning. We show below how it might be implemented in time $O\left(\left|O_{n c}\right|\right)$ where $\left|O_{n c}\right|$ is the sum of the size of all rows belonging to the overlap class.

The data structure we need must allow us to

1. split a part $C$ in $C^{\prime} C^{\prime \prime}$ in the number of the elements of $C$ touched;

2. add a new part to the left of to the right of the current partition in the number of the elements added;

3. test if the elements touched can be made consecutive;

4. test if a new row cut another one already embedded in the partition;

There might be many data structures implementation having these properties. We propose below a simple one. This structure can also replace that used in 22 for identifying all $\operatorname{Max}(X)$ used by Dahlhaus's algorithm (see Appendix B), and thus our whole algorithm only uses a single data structure.

We basically use an array of size $|\mathcal{C}|$ to store a stack which encodes a permutation of elements of $C$. Each cell of this array contains a column and a link to the part it belongs to. A part is coded as a pair of its beginning and ending positions in the array, relatively to the beginning of the array. A schematic representation of this data structure is given in Figure 3 .

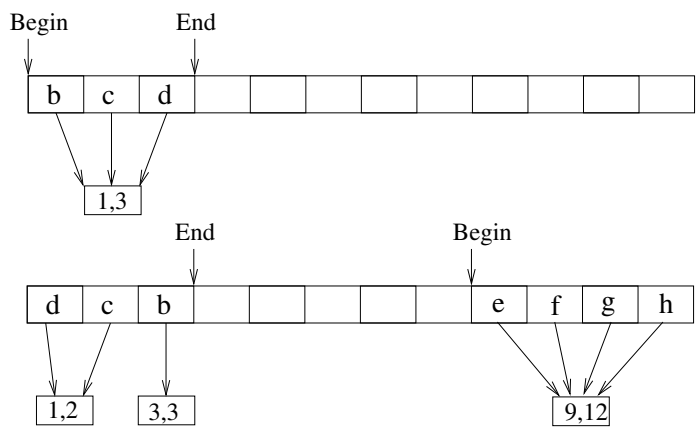

Fig. 3. Example continued: implementation of $(b c d)$ and then $(e f g h)(d c)(b)$ when refining $R_{2}=\{b, c, d\}$ by $R_{3}=\{c, d, e, f, g, h\}$.

Using this data structure, refining a part $C$ by one of its subset $C^{\prime \prime}$ can be easily done in $O\left(\left|C^{\prime \prime}\right|\right)$. Indeed, let $[i, j]$ be the bounds of $C$. We swap elements in the subtable $[i, j]$ to place all $s=\left|C^{\prime \prime}\right|$ elements of $C^{\prime \prime}$ at the end or at the beginning of this subtable as necessary. We then adjust the bounds of $C$ to $[i, j-s]$ or $[i+s, j]$ depending of the case and create a new set $[j-s+1, j]$ or $[i, i+s-1]$ on which the $s$ elements of $C^{\prime \prime}$ now point. 
Adding a new part to the left of to the right of the current partition in the number of the elements added is easy since it suffices to create a new part and move the pointers of the beginning or ending modulo $|\mathcal{C}|$. An example of such operation is shown in Figure 3 .

Assume that a new row $R$ used for refining cut a class in the partition $P$, and let $Y \subset R$ be the elements of $R$ that are already in the partition.

If $Y \neq R$, then, to verify $\mathrm{C} 1 \mathrm{P}$, all classes touched by $Y$ must be placed at an extremity of $P$, all parts from this extremity must be fully touched except the last one of which all elements touched has to be placed on the side of the extremity we considered. All these requirements can easily be checked in the number of elements of $R$, and if they are verified, a new part containing $R \backslash Y$ is added to the extremity.

If $Y=R$, then to verify $\mathrm{C} 1 \mathrm{P}$ there should be a left part that might not be fully touched followed by a series (that can be empty) of plenty touched parts and eventually a last part also not necessary fully touched. This is also not difficult to check in $O(|R|)$.

The novelty in our approach is that a new row $R$ might not cut the current partition, which has to be tested efficiently. This can also easily be checked in $O(|R|)$ on our structure. Indeed, it suffices to test if $R$ is included in a single part, in none, or contains all parts. We thus have:

Theorem 2. Testing the C1P of the rows belonging to a same overlap class can be done in $O\left(\left|O_{n c}\right|\right)$ time provided a swap overlap order $O_{n c}$ of it.

And eventually:

Corollary 1. Testing the C1P of a family $\mathcal{R}$ can be done in $O(|\mathcal{R}|)$ using a swap overlap order of each overlap class.

Proof. It suffices to compute all overlap classes of $\mathcal{R}$ using Algorithm 3 that provides for each overlap class a swap overlap order. Then Theorem 2 insures that $\mathrm{C} 1 \mathrm{P}$ can be tested on each overlap class in the number of rows belonging to this class. As overlap classes partition $\mathcal{R}$ and that $\mathcal{R}$ verifies $\mathrm{C} 1 \mathrm{P}$ if an only if each overlap class verifies $\mathrm{C} 1 \mathrm{P}$, the whole test can be done in $O(|\mathcal{R}|)$ time.

\section{References}

1. K.S. Booth and G.S. Lueker. Testing for the consecutive ones properties, interval graphs and graph planarity using pq-tree algorithm. J. Comput. Syst. Sci., 13:335379, 1976.

2. P. Charbit, M. Habib, V. Limouzy, F. de Montgolfier, M. Raffinot, and M. Rao. A note on computing set overlap classes. Information Processing Letters, 108(4):186$191,2008$.

3. T. Christof and J. Kececioglu. Computing physical maps of chromosomes with nonoverlapping probes by branch-and-cut. In RECOMB '99: Proceedings of the third annual international conference on Computational molecular biology, pages 115-123, 1999. 
4. E. Dahlhaus. Parallel algorithms for hierarchical clustering and applications to split decomposition and parity graph recognition. J. Algorithms, 36(2):205-240, 2000 .

5. M. Habib, R. McConnell, C. Paul, and L. Viennot. Lex-bfs and partition refinement, with applications to transitive orientation, interval graph recognition and consecutive ones testing. Theoretical Computer Science, 234:59-84, 2000.

6. B. Haeupler and R. E. Tarjan. Planarity algorithms via pq-trees (extended abstract). Electronic Notes in Discrete Mathematics, 31:143-149, 2008.

7. W.-L. Hsu. A simple test for the consecutive ones property. J. Algorithms, 43(1):1$16,2002$.

8. W.-L. Hsu and R. M. McConnell. PC-trees and circular-ones arrangements. Theoretical Computer Science, 296:99-116, 2003.

9. R. M. McConnell. A certifying algorithm for the consecutive-ones property. In SODA, pages 768-777, 2004.

10. T. Nishizeki and Md. S. Rahman. Planar Graph Drawing. World Scientific, 2004.

11. S. Young. Implementation of PQ-tree Algorithms. Master's thesis, University of Washington, 1977. A scan version is available at http://www.liafa.jussieu.fr/ raffinot/ftp/Young-PQ-tree.pdf 


\section{A Trace of Algorithm 3 on Our Example}

We trace below the recursive steps of Algorithm 3 on our example for the identification of the first overlap class while outputting the order $O_{1}=R_{2} R_{3} R_{4} R_{5} R_{7}$ $R_{3} R_{1} R_{9} R_{11}$.

1. Step 1. $O_{n c}=\varepsilon, n c=1$

2. $T I[2]$ is choosen in step 2 since it contains an interval of type $M$.

3. Step $3_{1}$. We consider $I_{1}=M 3=$ $\left[R_{2} R_{3}\right]$

4. $31_{1}$-(a) all occurrences of $M 3$ are removed out of $T I$

5. $3_{1}$-(b) $O_{1}=R_{2} R_{3}, N C[2]=1$, $N C[3]=1$

6. $3_{1}$-(c) Recursive call to Step $3_{2}$ on $R_{2}$ from $M 3$. We consider $I_{2}=M 5=$ $\left[R_{2} R_{3}\right]$

7. $3_{2}$-(a) all occurrences of $M 5$ are removed out of $T I$

8. $3_{2}$-(b) as $N C[2]=N C[3]=1$ no row si added to $O_{1}$

9. $33_{2}$-(c) Recursive call to Step $3_{3}$ on $R_{2}$ from $M 5$.

10. Entering Step $4_{3}$ since there is no more interval of type $M$ in $T I[2]$. We consider $I_{3}=E 4=\left[R_{4} R_{2}\right]$

11. $4_{3}$-(a) all occurrences of $E 4$ are removed out of $T I$

12. $4_{3}$-(b) Recursive call to Step $3_{4}$ on $T I[4]$. We consider $I_{4}=M 6=\left[R_{4} R_{5}\right]$

13. $3_{4}$-(a) all occurrences of $M 6$ are removed out of $T I$

14. $3_{4}$-(b) $O_{1}=R_{2} R_{3} R_{4} R_{5}, N C[4]=1$, $N C[5]=1$

15. $3_{4}$-(c) Recursive call to Step $3_{5}$ on $T I[4]$. As $T I[4]$ is now empty, we return to step $3_{4}$

16. $3_{4}$-(c) Recursive call to Step $3_{6}$ on $T I[5]$. We consider $I_{6}=M 7=$ $\left[R_{7} R_{5} R_{3}\right]$

17. $36^{-}$(a) all occurrences of $M 7$ are removed out of $T I$

18. $3_{6}$-(b) $O_{1}=R_{2} R_{3} R_{4} R_{5} R_{7}, N C[7]=$ 1

19. $3_{6}$-(c) Recursive call to Step $3_{7}$ on $R_{7}$ from $M 7$. We consider $I_{7}=E 2=$ $\left[R_{7} R_{1} R_{2} R_{9}\right]$

20. $4_{7}$-(a) all occurrences of E2 are removed out of $T I$
21. 47 -(b) Recursive call to Step $3_{8}$ on $T I[7]$. As $T I[7]$ is now empty, we return to step $4_{7}$

22. $4_{7}$-(c) $O_{1}=R_{2} R_{3} R_{4} R_{5} R_{7} R_{1} R_{9}$, $N C[1]=1, N C[9]=1$

23. $47_{-}$-(d) Recursive call to Step $3_{9}$ on $R_{1}$ We consider $I_{9}=E 1=\left[R_{11} R_{1}\right]$

24. 49 -(a) all occurrences of $E 1$ are removed out of $T I$

25. $4_{9}$-(b) Recursive call to Step $3_{10}$ on $R_{11}$ We consider $I_{10}=M 8=\left[R_{11} R_{9}\right]$

26. $310^{-}$(a) all occurrences of $M 8$ are removed out of $T I$

27. $3_{10-}$ (b) $O_{1}=R_{2} R_{3} R_{4} R_{5} R_{7} R_{1} R_{9} R_{11}$, $N C[11]=1$

28. 310 -(c) Recursive call to Step $3_{11}$ on $T I[11]$. As $T I[11]$ is now empty, we return to step 310

29. $33_{10}$-(c) Recursive call to Step 312 on $T I[9]$. As $T I[9]$ is now empty, we return to step $3_{10}$ than also ends, returning to Step 49

30. 49 -(c) Nothing to concatenate from $E 1=\left[R_{11} R_{1}\right]$ since the two rows are already in $O_{1}$.

31. 49 -(d) Recursive call to Step 313 on $T I[1]$. As $T I[1]$ is now empty, we return to step $4_{9}$ which also ends, thus returning to Step $4_{7-}$ (d)

32. 47 -(d) Recursive call to Step 314 on $T I[2]$. As $T I[2]$ is now empty, we return to step $4_{7}-(\mathrm{d})$

33. $4_{7-}$ (d) Recursive call to Step $3_{15}$ on $T I[9]$. As $T I[9]$ is now empty, we return to step $4_{7}$ which also ends, thus returning to Step $3_{6}$-(c)

34. $36_{6}$-(c) Recursive call to Step $3_{16}$ on $R_{1}$ from $M 7$. As $T I[1]$ is now empty, we return to step $3_{6}$-(c)

35. $3_{6}$-(c) Recursive call to Step $3_{17}$ on $R_{2}$ from $M 7$. As $T I[2]$ is now empty, we return to step $3_{6}$-(c)

36. $36_{6}$-(c) Recursive call to Step $3_{18}$ on $R_{9}$ from $M 7$. As $T I[9]$ is now empty, we 
return to step $3_{6}$-(c) which also ends, returning to Step $3_{4}$-(c)

37. $3_{4}$-(c) Recursive call to Step $3_{19}$ on $R_{3}$ from $M 7$. As $T I[3]$ is now empty, we return to step $33_{4}$-(c) which also ends, returning to Step $4_{3}$-(b)

38. $4_{3}$-(c) Recursive call to Step $3_{20}$ on $R_{2}$ from $E 4$. As $T I[2]$ is now empty, we return to step $3_{4}$-(c) which also ends, returning to Step $3_{2}$-(c).
39. $3_{2}$-(c) Recursive call to Step $3_{21}$ on $R_{3}$ from $M 5$. As $T I[3]$ is now empty, we return to step $3_{2}$-(c) which also ends, returning to Step $3{ }_{1}-(\mathrm{c})$.

40. $3_{1}$-(c) Recursive call to Step $3_{22}$ on $R_{3}$ from $M 3$. As $T I[3]$ is now empty, we return to step $33_{1}-(\mathrm{c})$ which also ends, ending the identification fo the first overlap class. The returning order for $n c=1$ is thus $O_{1}=$ $R_{2} R_{3} R_{4} R_{5} R_{7} R_{1} R_{9} R_{11}$.

\section{B Computing all $\operatorname{Max}(X)$}

In this appendix we recall the computation of $\operatorname{Max}(R)$ only slightly modified compared to the that published in 2 . The very small modifications is that we impose $\operatorname{Max}(R)$ to be greater or equal to $R$ in the $L R$ order, while in 2 the constraint for $\operatorname{Max}(R)$ is only to be of size greater or equal to that of $R$. This implies that in [2] and also in the original paper of Dahlhaus [4] $\operatorname{Max}(R)$ can be after $R$ in the $L R$ order if $|\operatorname{Max}(R)|=|R|$, which in fact complexifies the understanding of the algorithm.

We consider a boolean matrix BM of size $|\mathcal{F}| \times|\mathcal{C}|$ such that each row represents a set $R \in \mathcal{F}$ in the order of LR, and each column an element $c \in \mathcal{C}$. The value $\mathrm{BM}[i, j]$ is 1 if and only if $c_{j} \in R_{i}$.

Let us consider first below that all columns of $B M$ are lexicographically sorted. Figure 4 shows the $B M$ matrix for the set family of Figure 2 .

\begin{tabular}{|c|c|c|c|c|c|c|c|c|c|c|c|c|c|c|}
\hline & $\begin{array}{l}1 \\
\mathrm{a} \\
--\end{array}$ & $\begin{array}{l}2 \\
\mathrm{i}\end{array}$ & $\begin{array}{l}3 \\
1 \\
\end{array}$ & & k & $\begin{array}{l}6 \\
\text { b }\end{array}$ & $\begin{array}{l}7 \\
\mathrm{c}\end{array}$ & $\mathrm{d}$ & $\mathrm{h}$ & $\begin{array}{c}10 \\
\mathrm{f}\end{array}$ & & & left & right \\
\hline$x_{3}$ & 0 & 0 & 0 & 0 & 0 & 0 & 1 & 1 & 1 & 1 & 1 & 1 & 7 & 12 \\
\hline $\mathrm{X}_{9}$ & & 1 & 1 & 1 & 1 & 1 & 0 & 0 & 0 & 0 & 0 & 0 & 2 & \\
\hline $\mathrm{X}_{5}$ & & 0 & 0 & 0 & 0 & 0 & 0 & 0 & 1 & 1 & 1 & 1 & 9 & 12 \\
\hline$x_{2}$ & 0 & & & & & 1 & 1 & 1 & 0 & 0 & 0 & 0 & 6 & 8 \\
\hline$x_{1}^{2}$ & 1 & & & & & 1 & 0 & 0 & & & & 0 & 1 & 6 \\
\hline$x_{4}$ & 0 & & & & & 0 & & 1 & & 0 & 0 & 1 & 8 & 12 \\
\hline$X_{6}^{4}$ & & & & & & 0 & & 0 & 0 & 1 & 1 & 0 & 10 & 11 \\
\hline$x_{7}$ & & & & 0 & 0 & 1 & & & 1 & 0 & 0 & & 6 & 9 \\
\hline $\mathrm{X}_{8}$ & 1 & & 0 & 1 & 1 & 0 & & & 0 & & & & 4 & 5 \\
\hline$x_{10}$ & 0 & 0 & 1 & 0 & 1 & 0 & & & & & & & 3 & 5 \\
\hline $\mathrm{X}_{11}$ & 1 & 1 & 0 & 0 & 0 & 0 & 0 & 0 & 0 & 0 & 0 & 0 & 1 & \\
\hline
\end{tabular}

Fig. 4. Example continued: $B M$ matrix which lines are sorted in $L R$ order and which columns are sorted in lexicographic order.

For each $R \in \mathcal{F}$ we denote left $(R)$ (resp. $\operatorname{right}(R))$ the number of the column of $B M$ containing the leftmost (resp. rightmost) 1 in the row of $R$. 
Lemma 2 ([2]). Let $R_{1}, R_{2} \in \mathcal{F}$ such that $R_{2}$ overlaps $R_{1}$ in $B M$. Then there exists a row $R \leq_{L R} R_{2}$ such that $B M\left[R\right.$, left $\left.\left(R_{1}\right)\right]=0$ and $B M\left[R\right.$, right $\left.\left(R_{1}\right)\right]=1$.

Lemma $3([2])$. Let $R_{1} \in \mathcal{F}$. Then $\operatorname{Max}\left(R_{1}\right) \neq \emptyset$ if and only if there exists a row $R$ in $B M$ such that $B M\left[R, \operatorname{left}\left(R_{1}\right)\right]=0$ and $B M\left[R, \operatorname{right}\left(R_{1}\right)\right]=1$ and verifying $|R| \geq_{L R}\left|R_{1}\right|$.

Lemma 4 ([24]). Let $R_{1} \in \mathcal{F}$ such that $\operatorname{Max}\left(R_{1}\right) \neq \emptyset$. Then $\operatorname{Max}\left(R_{1}\right)$ corresponds to the highest row $R$ in $B M$ such that $B M\left[R, \operatorname{left}\left(R_{1}\right)\right]=0$ and $B M[R$, $\left.\operatorname{right}\left(R_{1}\right)\right]=1$.

Dahlhaus's approach for computing all $\operatorname{Max}\left(R_{1}\right)$ the smallest $R$ in $L R$ order such that $\mathrm{BM}\left[R, \operatorname{left}\left({ }_{1}\right)\right]=0$ and $\operatorname{BM}\left[R, \operatorname{right}\left(R_{1}\right)\right]=1$. Dahlhaus reduces the problem to LCA computations, which has been simplified in [2] using partitions.

Computing all $\operatorname{Max}(R)$ using set partitioning. We manipulate sorted partitions of $V$ that we refine by each $R \in \mathcal{R}$ taken in LR order, that is, in decreasing order of their sizes. The initial partition is the whole set $\mathcal{C}$ and denoted $P_{\mathcal{C}}$. The refinement is slightly restricted compared to that of Section 4 in the sense that $C$ is always split in $C^{\prime} C^{\prime \prime}$ (and never $C^{\prime \prime} C^{\prime}$ ) if $C^{\prime \prime}$ represents the set of elements in $R$. Refining a partition $P$ by a set $R \in \mathcal{R}$ consists in refining successively all parts in $P$. We note this refinement $\left.P\right|_{R}$.

For example (continued), if $P=\{a\}\{i, j, k, l\}\{b\}\{c, d\}\{e, f, g, h\}$ and $R=$ $R_{4}=\{d, e\},\left.P\right|_{R}=\{a\}\{i, j, k, l\}\{b\}\{c\}\{d\}\{f, g, h\}\{e\}$.

The approach requires 3 steps:

1. refine $P_{V}$ by all $R \in \mathcal{R}$ taken in LR order;

2. then compute for each $R \in \mathcal{R}$ the values of left $(R)$ and $\operatorname{right}(R)$ and sort all $R \in \mathcal{R}$ in a special order in regard with these values;

3. eventually refine $P_{V}$ again by all $R \in \mathcal{R}$ taken in LR order but using the informations computed in step 2 to compute all $\operatorname{Max}(R)$.

These 3 steps are detailed below.

Step 1 - Refining $P_{V}$. Let us consider the final partition we obtain after refining $P_{V}$ by each $R \in \mathcal{R}$ taken in $L R$ order. We note this partition $P_{f}$.

Lemma $5([2])$. The elements of $P_{f}$ are sorted accordingly to the lexicographical order of the columns of BM.

For example (continued), on the data in Figure 4. $P_{f}=\{a\}\{i\}\{l\}\{j\}\{k\}\{b\}$ $\{c\}\{d\}\{h\}\{f, g\}\{e\}$. Note that equal columns of $B M$ are in the same part of $P_{f}$ on which we fix an arbitrary order. 
Step 2 - Computing all left $(R)$ and right $(R)$ values. We then compute all $\operatorname{left}(R)$ and $\operatorname{right}(R)$ values on $P_{f}$. This can be done easily in $O(|\mathcal{R}|+n)$ time by scanning each $R \in \mathcal{R}$ and keeping the minimum and maximum position of one of its element in $P_{f}$. We also compute a data structure $A M$ that for each position $1 \leq i \leq|V|$ of $P_{f}$ gives a list of all $R \in \mathcal{R}$ such that $i=\operatorname{right}(R)$. All those lists are sorted in increasing order of left $(R)$. The structure also allows an element $R \in \mathcal{R}$ to be removed from the list $A M[\operatorname{right}(R)]$ in $O(1)$ time. This can be insured for instance using doubly linked list to implement each list, and the whole structure can easily be built in $O(n+m)$ time using bucket sorting.

Step 3 - Refining $P_{V}$ again and identifying all Max $(R)$. The main idea is the following. Assume that at a step of the refinement process in $L R$ order we refine a part $C=\left\{c_{i_{1}}, \ldots, c_{i_{k}}\right\}$ of a partition $P$ by $R_{2} \in \mathcal{R}$ and that it results two non empty parts $C^{\prime} C^{\prime \prime}$.

Lemma 6 ([2]). Let $R \in \mathcal{R}$ such that $|R| \leq\left|Y_{2}\right|$, left $(R) \in C^{\prime}$ and $\operatorname{right}(R) \in$ $C^{\prime \prime}$. Then $R_{2}=\operatorname{Max}(R)$.

The last phase of the algorithm thus consists in refining $P_{\mathcal{C}}$ again by all $R_{2} \in \mathcal{R}$ taken in $L R$ order. We first initialize all values $\operatorname{Max}(R)$ to $\emptyset$. Each time a new split $C^{\prime} C^{\prime \prime}$ appears (say between positions $l$ and $l+1$ ), for all $c \in C^{\prime \prime}$ all lists $A M[c]$ are inspected the following way: let $R$ be the top of one of those the list; while left $(R) \leq l, R$ is popped off the list and $\operatorname{Max}(R) \leftarrow R_{2}$. After having refined with $R_{2}, R_{2}$ is removed from the $A M$ structure.

Lemma 7 ([2]). The above algorithm correctly computes in 3 steps all Max $(R)$, $R \in \mathcal{R}$.

The partition refinement can be efficiently implemented using the data structure presented in Section 4 of that in 2] which is a simpler version of the first one.

Theorem 3 ([2]). The identification of all $\operatorname{Max}(R), R \in \mathcal{R}$, using partition refinement can be done in $\Theta(|\mathcal{R}|)$ time. 